Société d'histoire de la révolution de 1848 et des

révolutions du XIXe siècle

$56 \mid 2018$

Un autre $\mathrm{XIX}^{\mathrm{e}}$ siècle : I'Inde sous domination coloniale

\title{
Pour une histoire polycentrique des républicanismes atlantiques (années 1770 - années 1880)
}

\author{
Clément Thibaud
}

\section{OpenEdition}

\section{Journals}

Édition électronique

URL : http://journals.openedition.org/rh19/5593

DOI : $10.4000 /$ rh 19.5593

ISSN : $1777-5329$

Éditeur

La Société de 1848

Édition imprimée

Date de publication : 15 octobre 2018

Pagination : 151-170

ISSN : 1265-1354

\section{Référence électronique}

Clément Thibaud, «Pour une histoire polycentrique des républicanismes atlantiques (années 1770 années 1880) », Revue d'histoire du XIXe siècle [En ligne], 56 | 2018, mis en ligne le 15 octobre 2020, consulté le 05 janvier 2021. URL : http://journals.openedition.org/rh19/5593 ; DOI : https://doi.org/ $10.4000 /$ rh19.5593 


\section{CLÉMENT THIBAUD}

\section{Pour une histoire polycentrique des républicanismes atlantiques (années 1770 - années 1880)}

Un coup d'œil sur une mappemonde politique du XIX siècle appelle une conclusion lapidaire ${ }^{1}$ : la république y est avant tout une forme américaine de gouvernement ${ }^{2}$. Aujourd'hui, des États-Unis aux nations hispano-américaines en passant par Haïti, ces nations forment, à l'exception de la Suisse et de certaines républiques-cités européennes, les plus anciens kingless states du monde. Alors que la philosophie politique classique tenait pour évidente la faiblesse des républiques face aux monarchies, en raison des limites étroites de leurs territoires ou de leurs tendances à la division factionnelle, une douzaine de ces gouvernements s'établissent des Grands Lacs à la Terre de Feu au cours d'une courte période définie par l'Anglais Thomas Paine comme un "âge des révolutions». De la déclaration d'indépendance des États-Unis en 1776 à l'émancipation des républiques hispano-américaines au cours des années 1820, la carte politique du continent américain enregistre l'effondrement de la souveraineté monarchique dans d'immenses espaces gouvernés naguère par les empires européens. Après la parenthèse antimonarchique de 1848, l'établissement de la Troisième République en France, après 1870, précède l'effondrement de l'empire brésilien en 1889 et l'indépendance de Cuba à la fin du siècle, qui ferment cette séquence républicaine à l'échelle océanique.

Ce basculement renvoie à l'historiographie qui, dans les années 1950, repère pour la première fois une "Révolution atlantique» (Jacques Gode$\operatorname{chot}^{3}$ ) ou un "âge des révolutions démocratiques» (Robert R. Palmer ${ }^{4}$ ). Au lendemain de la Seconde Guerre mondiale, il s'agissait de dépasser les

1. Cette réflexion s'inspire des trois ateliers Southern Republicanism tenus de mai 2016 à septembre 2017 à Buenos Aires et Nantes, dans le cadre du projet STARACO (Université de Nantes), sous la direction de Gabriel Entin, Federica Morelli et moi-même, avec la participation d'une vingtaine de chercheurs.

2. Nous entendons ici, comme dans le reste de l'article, la notion d'Amérique dans son sens hémisphérique et non simplement nord-américain. Cette remarque vaut pour la sphère internationale "formalisée», exceptant les nombreuses communautés politiques non monarchiques des sociétés sans État reconnu.

3. Jacques Godechot, La grande nation. L'expansion révolutionnaire de la France dans le monde de 1789 à 1799, Paris, Aubier, 1956.

4. Robert R. Palmer, The Age of the Democratic Revolution. A Political History of Europe and America, 1760-1800, Princeton, Princeton University Press, 1959. 
approches nationales pour penser une séquence révolutionnaire européenne et nord-américaine - nord-atlantique -, en identifiant des causes communes à l'indépendance des États-Unis et aux révolutions d'Europe et de France. Ces approches, cultivées aussi par les ouvrages influents de Hannah Arendt ${ }^{5}$ et de Louis Hartz ${ }^{6}$, ont adopté un modèle de causalité à la fois unifié et séquentiel. Le repérage d'une matrice commune - le conflit entre la monarchie et les corps constitués pour Robert Palmer - expliquerait l'effet domino des révolutions à l'échelle océanique. Toujours cités aujourd'hui, ces livres ont eu le mérite de décloisonner une historiographie téléologiquement refermée sur la nation. Ils délaissaient cependant à la fois les enjeux coloniaux, la question de l'esclavage et ses acteurs, ignoraient la Révolution haïtienne, les indépendances ibéro-américaines et la réfraction de ces événements sur le continent africain. Leur description de la séquence révolutionnaire atlantique se fondait sur un impensé qui avait la force de l'évidence : la modernité nord-atlantique était le centre à partir duquel rayonnait l'invention révolutionnaire. En découlaient un schéma diffusionniste en cercles concentriques, et le déterminisme historiciste de l'expansion irrésistible de la Révolution atlantique.

Les grandes controverses du Republican Turn aux États-Unis ont transformé le débat historiographique sur la question. Elles ont marqué l'historiographie entre les années 1970 et 1990, en prêtant attention à la nature idéologique de la Révolution américaine. À en croire les œuvres fondatrices de Bernard Bailyn ${ }^{7}$, de John Pocock et de Gordon Wood ${ }^{8}$, et contrairement à ce qu' affirmait la doxa mémorielle, politique et historiographique du pays, les Founding Fathers n'avaient nullement souhaité fonder une société commerciale où devaient primer les droits individuels sur la puissance souveraine. Ils s'appuyaient au contraire sur une conception prescriptive de la liberté9, s'opposant à la dimension négative que lui donnait le libéralisme en isolant une sphère d'autonomie pour les individus et la société civile contre l'État ${ }^{10}$. L'héritage du tournant républicain imprègne encore aujourd'hui les études sur le républicanisme atlantique. Les concepts de "radicalisme whig" (Bernard Bailyn), d' "humanisme civique» (John Pocock) ou de "conception néo-romaine de la liberté» (Quentin Skinner) imposent un cadre théorique, méthodologique et géographique qui s’avère problématique lorsqu'il s'agit de

5. Hannah Arendt, On Revolution, New York, Viking Press, 1963, traduction française Essai sur la Révolution, Paris, Gallimard, 1967.

6. Louis Hartz, The Liberal Tradition in America. An Interpretation of American Political Thought since the Revolution, New York, Harcourt Brace and Co, 1955.

7. Bernard Bailyn, The Ideological Origins of the American Revolution, Cambridge (Mass.), Belknap Press of Harvard University Press, 1967, traduction française Les origines idéologiques de la Révolution américaine, Paris, Belin, 2010.

8. Gordon Wood, The Creation of the American Republic, 1776-1787, Chapel Hill, University of North Carolina Press, 1969.

9. Philip Pettit, Republicanism. A Theory of Freedom and Government, Oxford, Oxford University Press, 1999, traduction française Républicanisme. Une théorie de la liberté et du gouvernement, Paris, Gallimard, 2004, chapitres 1 et 2.

10. Isaiah Berlin, Two Concepts of Liberty, Oxford, Clarendon Press, 1966. 
penser l'histoire de la république à une échelle plus globale et polycentrique ${ }^{11}$. En plaçant la focale sur un espace de circulation allant de l'Italie aux Provinces-Unies, de la Grande-Bretagne aux Treize Colonies et aux États-Unis, le modèle «républicaniste» privilégie à la fois un centre géographique et une chronologie dont le cœur est l'Europe médiévale et moderne. Les acteurs de cette histoire sont des Européens ou leurs descendants outre-Atlantique. Malgré son exceptionnelle fécondité, en s'attachant avant tout aux concepts, dans une dimension certes atlantique mais en réalité anglo-américaine ${ }^{12}$, elle a laissé dans l'ombre d'autres mondes républicains marqués par des configurations de pensée et, surtout, des répertoires d'actions, des acteurs et des enjeux sociaux différents. C'est cette pluralité qui est aujourd'hui revendiquée par tout un ensemble de travaux.

Comment penser l'éclosion républicaine, de l'âge des révolutions à la fin du XVIII ${ }^{\mathrm{e}}$ siècle, dans une perspective susceptible de décrire l'ensemble du processus dans sa diversité et dans sa durée? De fait, l'ambition d'une histoire polycentrique des républiques de l'Atlantique pose des problèmes délicats. Comment le faire sans s'appuyer sur l'idée d'un modèle républicain essentialisé, qui se déploierait dans le temps et dans l'espace? L'idée même d'une séquence républicaine se révèle problématique, en ce qu'elle favorise les explications déterministes ou téléologiques. Elle pose également d'épineuses questions de méthode dès lors qu'il s'agit de rendre compte à la fois de l'unité du processus et de la singularité de ses déclinaisons locales. La tâche impose, d'abord, un travail descriptif visant à faire un relevé des moments antimonarchiques à petite échelle, pour essayer de définir les liens qui, éventuellement, les associent.

Plusieurs thèses seront ici défendues. La centralité américaine du républicanisme au XIX' siècle n'est pas seulement un fait géographique ${ }^{13}$. Elle est liée à la double nature impériale et coloniale de ces sociétés à l'époque moderne : les répertoires d'idées et d'actions antimonarchiques aboutissent à des régimes durables qui rejettent davantage l'empire que les rois. L'esclavage et son abolition, de même que l'égalité civile des sujets coloniaux dégradés en droit - libres de couleur et Amérindiens - constituent, de ce fait, autant d'enjeux structurants de la séquence républicaine atlantique ${ }^{14}$. Cette particularité anti-impériale explique, on le verra, l'association précoce du républicanisme au droit naturel et au libéralisme - un attelage que ne permettent guère de penser les coordonnées historiographiques posées par le Republican

11. À l'exception, peut-être, de Quentin Skinner.

12. Ce n'est pas le cas, il est vrai, de Martin van Gelderen et Quentin Skinner (eds), Republicanism. A Shared European Heritage, Cambridge, Cambridge University Press, 2002, 2 volumes.

13. Au sens continental.

14. En France, l'abolition de l'esclavage et l'adoption du suffrage universel masculin, en 1848, réunissent de manière spectaculaire les deux enjeux. Il faut remarquer cependant que beaucoup de républicains et de libéraux peuvent parfaitement justifier l'esclavage et la hiérarchie des races, non comme une exception à leurs idéaux, mais en cohérence avec ceux-ci. 
Turn. Une autre question concerne les effets retour des républicanismes libéraux des Américains du Nord et du Sud vers l'Europe et la Méditerranée dès les années 1820. L'exigence constitutionnelle et libérale, partagée par une multiplicité d'acteurs opposés à l'Europe de la légitimité, encourage la création d'un espace de circulation cosmopolite, de part et d'autre de l'océan.

$\mathrm{Si}$, au cours de ces vingt dernières années, beaucoup de travaux ont éclairé ces problèmes, il reste beaucoup à faire pour dessiner une image globale du processus de républicanisation à l'échelle de l'hémisphère américain, en incluant sa partie méridionale, sans parler de l'Atlantique (et du reste). Cet article n'a pas la prétention de combler cette lacune : son objet est plutôt de dessiner une carte des relations, d'évoquer les voies possibles pour rendre compte de celles-ci, et de suggérer des rapprochements entre des lignes historiographiques demeurées trop souvent parallèles.

\section{LES GÉOGRAPHIES PLURIELLES DU RÉPUBLICANISME}

La républicanisation du continent est, par définition, une histoire atlantique, comme en attestent déjà les causes et le cours de la Révolution américaine qui ouvre le cycle. $\mathrm{Si}$, en 1792, la chute de la monarchie française ne doit rien aux revendications anti-impériales ou anticoloniales, elle bouleverse l'ordre politique, la culture et la société caribéenne avec le décret de liberté générale, un processus qui se répète avec la Seconde République en 1848. Les révoltes de Saint-Domingue et l'indépendance d'Haïti sont bien évidemment imbriquées dans la Révolution de la métropole ${ }^{15}$. Les républiques-sœurs, aux Pays-Bas et dans la péninsule italienne, ont, elles aussi, un écho atlantique et global $^{16}$. La révolte de l'Irlande, en 1798 , soutenue par le Directoire, produisit un contingent de proscrits qui combattit ensuite sur les deux rives de l'Atlantique. Les indépendances hispano-américaines créent un vaste espace républicain, allant de la Californie à Buenos Aires et Santiago du Chili, après les années 1820 .

Il faut inclure également dans ce relevé factuel les mouvements antimonarchiques - ou plutôt anti-impériaux - qui n'ont pas prospéré. Des Canadiens français adoptent ainsi la bannière républicaine dans le Haut et le Bas-Canada pour contester la tutelle britannique en 1836-1838 ${ }^{17}$. À Cuba, demeurée espagnole, un mouvement annexionniste, favorable à l'intégration

15. Bernard Gainot, La révolution des esclaves. Haïti, 1763-1803, Paris, Vendémiaire, 2017.

16. Pierre Serna [dir.], Républiques soeurs : le Directoire et la révolution atlantique, Rennes, Presses universitaires de Rennes, 2009. Les Provinces-Unies étaient une République impériale avant d'être satellisées dans l'orbite de la France révolutionnaire : Wim Klooster et Gert Oostindie [dir.], Curaçao in the Age of Revolutions, 1795-1800, Leiden, KITLV Press, 2011, et Annie Jourdan, La révolution batave entre la France et l'Amérique, 1795-1806, Rennes, Presses universitaires de Rennes, 2015.

17. Michel Ducharme, Le concept de liberté au Canada à l'époque des révolutions atlantiques, 1776-1838, Montréal, McGill-Queen’s University Press, 2010. 
de l'île aux États-Unis, se développe au cours de la décennie $1850^{18}$. Pendant la guerre de Dix ans (1868-1878), l'armée des mambises se bat contre les troupes venues de la métropole au nom d'une république qui effacerait la race et l'esclavage ${ }^{19}$. Royaume puis empire, le Brésil est assailli par des rébellions antimonarchiques. Celles-ci arment les revendications d'élites locales qui s'allient à des groupes populaires en lutte pour la démocratisation du gouvernement et l'égalité des droits entre blancs et non-blancs. En attestent la Révolution de Pernambouc (1817), la fugace Confédération de l'Équateur $(1824)^{20}$, ou la plus durable République du Rio Grande do Sul (1836$1845)^{21}$, sans oublier de nombreuses autres rébellions anti-impériales ${ }^{22}$. En 1888, l'abolition de l'esclavage précède l'installation, un an plus tard, d'une République dont les élites se réclament du positivisme.

Le continent africain participe à cette conjoncture océanique. S'il conviendrait, dans un tout autre registre, de prendre en compte les communautés sans roi du Congo, il faut mentionner la fondation du Liberia, en 1822, par la New Colonization Society, dans le but d'accueillir les esclaves émancipés aux États-Unis. La colonie, mêlant natifs et affranchis, devient un commonwealth indépendant en $1847^{23}$. Dans une autre logique, anti-impériale cette fois-ci, des républiques boers s'établissent contre la conquête britannique en 1795 . Après le Grand Trek émergent l'État libre d'Orange (1854) et la République du Transvaal (1857). Dans l'empire français, le renversement des rois en 1792, puis en 1848, entraine la républicanisation des Quatre-Communes du Sénégal, avec de profondes implications locales lors des deux abolitions de l'esclavage ${ }^{24}$.

Où placer la césure entre l'âge des révolutions atlantiques, marqué par l'enjeu du régime politique, et une autre périodisation révolutionnaire, où prime la transformation de la société? Bien qu'essentiellement européen, le moment 1848 représente peut-être la dernière étape d'une séquence révolutionnaire à l'échelle de l'Atlantique et du monde. C'est du reste ce que suggère

18. Romy Sánchez, Quitter la Très Fidèle. Exilés et bannis au temps du séparatisme cubain, 18341879, thèse d'histoire sous la direction d'Annick Lempérière, Université Paris 1 Panthéon-Sorbonne, 2016.

19. Ada Ferrer, Insurgent Cuba. Race, Nation, and Revolution, 1868-1898, Chapel Hill, University of North Carolina Press, 1999, chapitre 1.

20. Luiz Geraldo Silva, «Pernambuco y la independencia : entre el federalismo y el unitarismo», Nuevo Mundo Mundos Nuevos, consulté le 12 décembre 2017 (DOI : 10.4000/nuevomundo.64766).

21. Moacyr Flores, Modelo politico dos Farrapos: as idéias politicas da Revolução Farroupilha, Porto Alegre, Editora Mercado Aberto, 1978.

22. Comme la Fédération de Guanais, la Sabinada, Cabanagem, la Balaiada. Cf. José Murilo de Carvalho, A formação das almas: o imaginário da república no Brasil, Sáo Paulo, Cia. das Letras, 1990 ; Celia Freire A. Fonseca, "Lidée républicaine au Brésil», Annales historiques de la Révolution française, 1994, volume 298, no 1, p. 715-726.

23. Eric Burin, Slavery and the Peculiar Solution. A History of the American Colonization Society, Gainesville, University Press of Florida, 2008.

24. Les études manquent sur ces périodes. $C f$. Ibrahima Thioub, "Stigmates et mémoires de l'esclavage en Afrique de l'Ouest: le sang et la couleur de peau comme lignes de fracture", FMSH-WP-2012-23. 2012.<halshs-00743503, consulté le 11 avril 2018. 
Janet Polasky dans son dernier livre, Revolution without Borders ${ }^{25}$. L'historienne étatsunienne distingue un premier cycle révolutionnaire, embrassant les révolutions de l'Amérique du Nord et de l'Europe jusqu'à l'indépendance haïtienne (1776-1804), et un second qui commence avec la chute des empires ibériques et finit avec le Printemps des Peuples (1808-1848). Cette coupure interroge, cependant, puisque les révolutions de Terre-Ferme - celles de Bolívar - articulent révolutions haïtienne et hispaniques ${ }^{26}$. Convient-il de prolonger jusqu'à la fin du XIX ${ }^{\mathrm{e}}$ siècle cette séquence atlantique, jalonnée par la Seconde République française, les éphémères républiques de l'Italie (18481849) et de l'Espagne (1873-1874) ? La consolidation du régime, en France et au Brésil au cours des années 1880 va de pair avec son renforcement dans les républiques américaines, fournissant à cette histoire un terminus ad quem crédible.

Malgré tout ce qu'ont pu dire ou écrire ses promoteurs, cette séquence républicaine n'a pas eu le caractère irrésistible d'un processus nécessaire. Les moments de reflux ont marqué les années 1860 durant lesquelles certaines puissances européennes ont tenté de reprendre pied sur le continent, avec la reconquête fugace, par l'Espagne, de Santo Domingo et des îles Chinchas, au large du Pérou, ou encore l'intervention française au Mexique ${ }^{27}$. Les ÉtatsUnis se sont comportés en République impériale vis-à-vis des Amérindiens - et des Mexicains - dans leur poussée vers l'Ouest, de même que l'Argentine et le Chili à la fin du siècle à l'égard des populations amérindiennes. Ces républiques, faut-il le rappeler, demeuraient profondément inégalitaires en raison de leur héritage colonial, d'abord ébranlé par les répertoires de la liberté politique mais bientôt renforcé, après 1860, par de nouvelles conceptions raciales.

Nonobstant, ce catalogue de "faits atlantiques", petits et grands, confirme notre observation initiale : le continent américain, jusqu'en 1870 au moins, est l'épicentre du républicanisme dans le monde. L'Europe incarne, aux yeux des Américains du Nord et du Sud, un continent monarchique et conservateur, malgré la parenthèse célébrée de 1848 , suivie par la déception du coup d'État de $1851^{28}$. Les temps faibles du républicanisme européen doivent être pensés en lien avec ses moments forts en Amérique hispanique et aux États-

25. Janet Polasky, Revolutions without Borders. The Call to Liberty in the Atlantic World, New Haven, Yale University Press, 2015, introduction.

26. Alejandro E. Gómez Pernía, Le spectre de la révolution noire. L’impact de la révolution haïtienne dans le monde atlantique, 1790-1886, Rennes, Presses universitaires de Rennes, 2013; Juan Francisco Martínez Peria, Lazos revolucionarios: Influencias, encuentros y desencuentros entre Haití, Venezuela y Nueva Granada en la época de la Independencia (1789-1830), doctorat de l'Universitat Pompeu Fabra, 2015; Frédéric Spillemaeker, «Quand les cocardes étaient marronnes : La Trinité espagnole en révolution", Monde(s), 2017, $\mathrm{n}^{\circ} 12$, p. 221-237.

27. Guillermo Palacios et Erika Pani [dir.], El poder y la sangre : guerra, estado y nación en la década de 1860, Mexico, Fondo de Cultura Económica, 2014.

28. James E. Sanders, The Vanguard of the Atlantic World. Creating Modernity, Nation, and Democracy in Nineteenth-Century Latin America, Durham, Duke University Press, 2014. La GrandeBretagne échappe souvent à la critique (merci à Romy Sánchez pour cette remarque). 
Unis, et mis en relation avec des espaces a priori extérieurs à la problématique, comme certains États côtiers de l'Afrique ou le Brésil. Ce changement d'échelle ouvre la perspective d'une histoire polycentrique de l'Atlantique politique, dont la trame circulatoire s'est construite dans l'expansion impériale et reconfigurée à l'âge des révolutions. La densité et l'ancienneté de ces liens expliquent la pertinence d'une telle échelle et d'une telle démarche depuis l'époque des Lumières et des révolutions jusqu'au triomphe du nationalisme, à la fin du XIX ${ }^{e}$ siècle, et bien sûr au-delà.

Il convenait d'ébaucher une géographie et une chronologie avant de préciser ce qu'on entend ici par république et républicanisme. Ces notions renvoient à des répertoires intellectuels et à des expériences politiques si anciennes qu'elles ont revêtu, avec le temps, un caractère polysémique faisant obstacle à la compréhension de ce qu'elles sont censées décrire. Rappelons qu'au XIX ${ }^{\mathrm{e}}$ siècle encore, le républicanisme ne s'oppose pas toujours à la monarchie, puisqu'il ne désigne pas nécessairement une forme de régime. Sur ce point, la philosophie politique classique, d'Aristote à Montesquieu, a préféré s'appuyer sur une autre distinction que celle de la royauté et de la république, en distinguant trois types de gouvernements : ceux de l'un, des meilleurs et des plus nombreux, soit grosso modo, avec des variations dans le temps, monarchie, aristocratie et démocratie. Ainsi la république-régime décrit-elle l'un des trois spectres de sens qu'a revêtu le concept dans l'histoire, mais cette acception fut loin d'être hégémonique à l'époque moderne. Dans la plupart des traditions, la république désigne un répertoire de valeurs avant tout politiques et morales, insistant sur les qualités que tout citoyen doit pouvoir cultiver dans une cité vertueuse et bien ordonnée. C'est cette dimension axiologique que privilégie le paradigme «républicaniste» de l'école de Cambridge. Au XVIII ${ }^{e}$ siècle, ce type de discours est repérable dans toutes les grandes monarchies européennes et leurs prolongements impériaux, trouvant une expression artistique dans le néo-classicisme. Il reste vivace au $\mathrm{XIX}^{\mathrm{e}}$ siècle.

La troisième acception est fondée sur la notion de communauté civique, quelle que soit la forme de l'État. La république-communauté décrit les caractères sociaux et territoriaux de la république en tant que cité. Elle renvoie au débat ancien sur la bonne taille de la cité, ouvert par Aristote puis revivifié après Montesquieu ${ }^{29}$. Elle importe particulièrement dans le passage des monarchies aux républiques qui voit s'effondrer la centralité royale, posant, par contrecoup, la question de l'articulation politique de l'État aux niveaux local et régional. En Amérique, la souveraineté revient aux provinces et aux villes, qui doivent se fédérer pour forger l'unité. De sorte que le fédéralisme représente l'un des enjeux fondamentaux des nouvelles nations. La

29. Judith N. Shklar, «Montesquieu and the new republicanism», in Gisela Bock, Quentin Skinner et Maurizio Viroli (eds), Machiavelli and Republicanism, Cambridge, Cambridge University Press, 1991, p. 265-280. 
république composée apparaît comme la solution idéale pour faire vivre les libertés locales, tout en constituant un front commun face à l'empire unitaire, forcément liberticide ${ }^{30}$. Davantage que le suffrage, le fédéralisme radical apparaît comme le régime démocratique par excellence des communautés sans roi.

\section{Penser l'ÂGe des RÉvolutions atlantiques : QUESTIONS DE MÉTHODE}

Comment penser l'unité des révolutions atlantiques, ou, du moins, leur articulation? La perspective comparée, tout d'abord, présente le mérite de rendre compte de chacune d'elles à partir de son propre contexte d'émergence. Le livre pionnier de Lester D. Langley a ouvert la voie ${ }^{31}$, renouvelé par Wim Klooster plus récemment ${ }^{32}$, mais cette démarche, critiquée par la "nouvelle histoire atlantique», n'est pas celle qui est la plus pratiquée aujourd'hui. L'approche contextuelle, mise en avant dans un article récent par Nathan Perl-Rosenthal ${ }^{33}$, s'attache à la description des références et des pratiques culturelles communes aux différentes sociétés de l'Atlantique pour expliquer la commensurabilité des processus républicains. Elle importe ici pour comprendre à la fois l'unité chronologique des révolutions atlantiques et la similarité de leurs conséquences politiques, avec la souveraineté populaire, le gouvernement représentatif et constitutionnel, la citoyenneté. Si cet Atlantique décrit un espace où se déploient certains paradigmes communs, c'est parce qu'il a été dessiné par des trames socio-économiques et des échanges politiques de longue durée, reliant le continent américain à l'Europe et à de vastes parties de l'Afrique depuis le XVIe siècle. Cet espace atlantique a été forgé par la construction des empires européens, la traite des esclaves, le commerce colonial ${ }^{34}$. Mêlant populations amérindiennes, africaines et européennes, les sociétés ultramarines sont à la fois impériales, en ce qu'elles prolongent et continuent l'Europe, et coloniales, du fait qu'elles fabriquent de nouvelles formes de hiérarchie sociale avec la conquête - et parfois l'incorporation - des peuples autochtones et les migrations forcées venues du continent africain. La pertinence de l'échelle atlantique, par ailleurs discutable et discutée, est liée à la présence nombreuse d'une population

30. Peter S. Onuf et Nicholas G. Onuf, Federal Union, Modern World. The Law of Nations in an Age of Revolutions, 1776-1814, Madison, Madison House, 1993.

31. Lester D. Langley, The Americas in the Age of Revolution, 1750-1850, New Haven, Yale University Press, 1996.

32. Wim Klooster, Revolutions in the Atlantic World. A Comparative History, New York, New York University Press, 2009.

33. Nathan Perl-Rosenthal, "Atlantic cultures and the age of revolution", The William and Mary Quarterly, volume 74, $\mathrm{n}^{\circ}$ 4, 2017, p. 667-696.

34. Les grands ouvrages de l'école des Annales ont décrit cette construction d'un Atlantique économique, social et politique, notamment Pierre Chaunu, Séville et l'Atlantique (1504-1650), Paris, Armand Colin, 1955. 
d'origine européenne et africaine transplantée sur le continent américain. Cela a bâti un espace intégré, dont la densité des connexions intercontinentales était supérieure à celle des mondes coloniaux de l'océan Indien et de l'Insulinde par exemple.

Si l'approche contextualiste permet de rendre compte de la "séquence» des révolutions atlantiques sans l'assimiler à un enchaînement de causes et d'effets nécessaires, elle risque d'homogénéiser les contextes des différents espaces et sociétés de l'Atlantique pour servir son propos. Une histoire connectée des révolutions républicaines de l'Atlantique pourrait éviter cet écueil. Est-elle pour autant possible? Le chantier est ouvert, en commençant par les relations les plus évidentes, à l'intérieur d'un même empire ou à l'échelle d'une région aux souverainetés enchevêtrées, comme la Caraïbe. Le propos consiste ici à éclairer les relations entre les différents processus révolutionnaires à partir des liens concrets qui ont pu les articuler : mobilités de personnes, diffusion de pratiques politiques et des répertoires d'idées sur fond de trafics commerciaux licites ou interlopes. Ces enquêtes supposent l'association de la microhistoire et de la petite échelle d'analyse. Visant à dépasser les métaphores de la connexion, du transfert ou de la diffusion de modèles, cette histoire connectée reste encore largement à écrire. Elle n'est du reste pas envisageable pour tous les moments révolutionnaires. Pour des raisons évidentes de chronologie, la Révolution américaine n'est pas susceptible d'un tel traitement, sauf à la rapporter aux deux révolutions anglaises $\mathrm{du} \mathrm{XVII}^{\mathrm{e}}$ siècle $^{35}$. Elle court également le risque de reproduire la structure téléologique et occidentalocentrée des perspectives comparatistes ou séquentielles. Elle offre pourtant la possibilité de penser le cycle révolutionnaire de l'Atlantique comme un rhizome, en insistant sur la réappropriation et la traduction locale des circulations révolutionnaires ${ }^{36}$. Le risque est cependant de négliger aussi bien la singularité de chacun des moments révolutionnaires que leur caractère disruptif. À condition de ne pas en faire un outil qui prétendrait expliquer le tout des révolutions et de leurs relations, l'approche connectée peut éclairer les rapports entre moments républicains. Sans qu'elle soit théorisée comme telle, elle a permis d'enrichir l'interprétation de la jeune république américaine ${ }^{37}$, des révolutions hispano-américaines et de l'indépendance du Brési ${ }^{38}$, en insérant ces processus dans une trame transnationale qui leur donnent sens. Le maître mot, en anglais, est celui d'entanglement,

35. Bernard Bailyn, The Ideological Origins..., op. cit.

36. Je me permets de renvoyer à Libérer le Nouveau Monde. La fondation des premières républiques hispaniques (Colombie et Venezuela, 1780-1820), Mordelles, Les Perséides, 2017, chapitre 2.

37. Ashli White, Encountering Revolution. Haiti and the Making of the Early Republic, Baltimore, Johns Hopkins University Press, 2012.

38. Voir le travail séminal de João Paulo G. Pimenta, Brasil y las independencias de Hispanoamérica, Castellón, Publicacions de la Universitat Jaume I, 2007, et Id., Estado y nación hacia el final de los imperios ibéricos. Río de la Plata y Brasil, 1808-1828, Buenos Aires, Sudamericana, 2011. 
qui désigne l'intrication de plusieurs processus révolutionnaires et offre des pistes prometteuses de recherche ${ }^{39}$.

De nombreuses questions se font jour. Comment identifier les contextes communs, les liens entre ces moments républicains? Comment évaluer et hiérarchiser la force de leurs effets? Ces interrogations travaillent l'historiographie des révolutions atlantiques et des cultures politiques, républicaines ou non, qui en furent le produit. L'enquête porte notamment sur la politisation de l'espace impérial, et, au-delà, atlantique. Certains suivent la piste des "papiers séditieux", la diffusion des livres ou bien encore la trajectoire des rumeurs. D'autres s'attachent au travail des traducteurs ou bien à la mobilité des rebelles, militaires ou exilés. D’autres, encore, cherchent à identifier la construction de sphères publiques transnationales, ou bien à suivre la transmission des pratiques révolutionnaires. Les possibilités sont nombreuses et les choix d'objet colorent des options historiographiques fortes où l'histoire sociale "par en bas", l'histoire culturelle du politique, l'histoire du droit ou l'histoire intellectuelle, inspirées ou non par les études subalternistes ou postcoloniales, incarnent des positions qui ne sont pas toujours inconciliables.

Si l'on s'en tient aux conditions contextuelles, une thèse courante dans l'historiographie américaine fait ainsi de l'existence supposée d'une société civile d'individus avant l'indépendance la condition première de la Révolution américaine. Cette analyse n'est toutefois valable que pour les citoyens de l'ancien et du nouveau régime, c'est-à-dire les Blancs. D'autres suggèrent le caractère moderne, avant la lettre, du monde colonial américain, en soulignant les dimensions capitalistes de la plantation esclavagiste ${ }^{40}$. Dans le monde hispanique, les éléments de continuité entre ancien et nouveau régime relèveraient moins de la modernité que du caractère corporatif de la Couronne espagnole. L'historien Manuel Herrero y voit une «monarchie de républiques ", ces dernières étant conçues comme autant de pouvoirs urbains étendus à leur plat pays à travers le pouvoir juridictionnel ${ }^{41}$. C'était déjà la proposition d'Annick Lempérière dans son étude pionnière sur la ville de Mexico $^{42}$. Ce républicanisme d'Ancien Régime, présent dans les riches villes italiennes ou aux Pays-Bas, n'est pas seulement européen. À l'abri d'une

39. Fabrício Prado, Edge of Empire. Atlantic Networks and Revolution in Bourbon Rio de la Plata, Berkeley, University of California Press, 2015; Ada Ferrer, Freedom's Mirror. Cuba and Haiti in the Age of Revolution, New York, Cambridge University Press, 2014.

40. Trevor Burnard, John Garrigus, The Plantation Machine. Atlantic Capitalism in French SaintDomingue and British Jamaica, Philadelphie, University of Pennsylvania Press, 2016.

41. Manuel Herrero Sánchez [dir.], Repúblicas y republicanismo en la Europa moderna (siglos XVI-XVIII), Madrid, Fondo de Cultura Económica, 2017; Gabriel Entin, La République en Amérique hispanique: langages politiques et construction de la communauté au Rio de la Plata, entre monarchie catholique et révolution d'indépendance, thèse d'histoire sous la direction de Pierre Rosanvallon, EHESS, 2011.

42. Annick Lempérière, Entre Dieu et le roi, la république. Mexico, XVI -XIXe siècles, Paris, Les Belles Lettres, 2004. Pour le Brésil, João Luís Ribeiro Fragoso, Maria Fernanda Bicalho et Maria de Fátima Gouvêa, O Antigo Regime nos trópicos: a dinâmica imperial portuguesa, séculos XVI-XVIII, Rio de Janeiro, Civilização Brasileira, 2010. 
noblesse qui se fait discrète dans l'hémisphère, sauf peut-être en NouvelleEspagne et au Pérou, il s'épanouit dans les cités où les lignées d'origine européenne se partagent le pouvoir municipal. Ces communautés politiques locales et provinciales jouent un rôle fondamental dans le basculement républicain des empires de l'Atlantique ${ }^{43}$.

\section{EMPIRE, RÉPUBLIQUE ET LIBÉRALISME}

Dans le monde américain, les républiques sont donc nées au cours de guerres d'indépendance contre des empires. Au sein des élites coloniales, les revendications économiques, en particulier commerciales, ont joué un rôle important dans le déclenchement ou la consolidation du processus séparatiste dès la fin du XVIIIe siècle. Si toutes n'étaient pas libre-échangistes ni même impliquées dans les circulations au long cours, elles souhaitaient pouvoir commercer plus librement et à de meilleurs conditions douanières dans le cadre de l'exclusif et, si possible, au-delà. La guerre de Sept ans a déclenché une série de réformes militaires et fiscales au sein de toutes les monarchies de l'Atlantique. Pour la Grande-Bretagne et l'Espagne, ce fut l'occasion de définir une politique impériale plus systématique et consciente d'elle-même, et d'en changer la nature. Cette ambition a suscité la critique d'une dissymétrie "coloniale» chez nombre de Blancs. C'est cette dimension de l'articulation coloniale l'inégalité entre métropoles et colonies - que visent les revendications autonomistes et bientôt républicaines. Ces dernières sont portées avant tout par des Européens d'outre-mer qui défendent, pour eux-mêmes, la conception impériale d'une monarchie égale en ses parties ${ }^{44}$. Cela conduit parfois à taire la différence coloniale qu'incarnent pourtant l'esclavage et les statuts racialisés. Ces demandes s'articulent très vite à une exigence constitutionnelle, parfois ancienne, qui garantirait les droits des Européens d'outre-mer à égalité avec ceux des métropoles, aux dépens des inégaux et des esclaves. Ce schéma est valable pour les Treize Colonies britanniques, la Saint-Domingue des colons, l'Amérique hispanique (et même le Brésil en 1820) : la demande d'égalité entre provinces métropolitaines et ultramarines s'accompagne d'une revendication constitutionnelle, dont le rejet débouche sur l'indépendance ${ }^{45}$. Les républiques américaines, de ce fait, sont travaillées dès l'origine par ce qu'on nommera commodément le «libéralisme ${ }^{46}$ ", avec la question des droits des

43. José Maria Portillo Valdés, Crisis atlántica : autonomía e independencia en la crisis de la monarquia hispana, Madrid, Marcial Pons, 2006.

44. Pauline Maier, From Resistance to Revolution. Colonial Radicals and the Development of American Opposition to Britain, 1765-1776, New York, W.W. Norton, 1991.

45. Sauf au Brésil.

46. La doctrine libérale, dans sa cohérence, n’est formulée qu’à la fin du XIX'e siècle : Duncan Bell, Reordering the World. Essays on Liberalism and Empire, Princeton, Princeton University Press, 2016, chapitre 2. Pour le monde ibérique, $c f$. Javier Fernández Sebastián [dir.], La aurora de la libertad: los primeros liberalismos en el mundo iberoamericano, Madrid, Marcial Pons, 2012. 
individus, du gouvernement représentatif et de la liberté économique. Audelà, c'est même l'enjeu démocratique qui se dessine, en lien avec l'épineuse (re)définition de la citoyenneté.

En critiquant la dissymétrie impériale entre centre et périphéries, ces révolutions fragilisent en effet l'autre dimension de l'articulation coloniale : la hiérarchie des statuts, des qualités et des conditions, le plus souvent racialisées, qui structurent la société. L'exigence constitutionnelle ouvre un champ de débats et de combats, concernant l'exclusion ou l'inclusion des différents types d'habitants dans le cercle de la citoyenneté nouvelle. La plupart demeurent des républiques esclavagistes, et même des communautés "raciales», comme les États-Unis ou les républiques boers, alors que l'Amérique hispanique choisit d'effacer constitutionnellement la macule de la «race», sans abolir pour autant le statut servile, ni se débarrasser des préjugés contre les métis et les Amérindiens. Il n'est donc pas exagéré de dire que ces mouvements révolutionnaires, devenus républicains, se construisent contre des empires en voie de "colonialisation", plutôt qu'en opposition à des monarchies. Avant le processus indépendantiste, les fondements de la royauté sont rarement mis en cause. La religion joue un grand rôle dans la protection de l'institution monarchique : c'est l'une des grandes difficultés qu'eurent à affronter les patriotes sud-américains par exemple ${ }^{47}$.

Les républiques les plus durables issues de l'âge des révolutions se sont opposées à l'empire colonial, ou plutôt à certains aspects de celui-ci. Il s'agit, en réalité, d'un fait de longue durée qui éclaire l'histoire européenne et questionne la nature même des premiers républicanismes américains. On peut les décrire comme des régimes de l'égalité civile et de la participation civique, où l'enjeu de la transmission généalogique des statuts et des conditions s'avère essentiel comme fondement, ou non, de droits individuels et collectifs. Alors pourquoi le régime a-t-il fleuri dans des sociétés où ces valeurs contredisaient point par point l'expérience que chacun pouvait faire de l'ordre social? Si les répertoires républicains, articulés ou non au «libéralisme " politique ou économique, furent mobilisés pour penser l'esclavage, son abolition et la citoyenneté des individus dégradés en droit, il faut remarquer que ces États reconduisirent, en les transformant, bien des hiérarchies et des statuts coloniaux, en dépit de la reconnaissance de droits naturels. C'est sans doute parce que la notion d'égalité s'y révélait si problématique qu'elle fut politisée de manière précoce. La synthèse libérale républicaine, en prônant le retour à une nature supposée de l'homme, permettait de justifier à nouveaux frais les inégalités de fait ou de droit entre individus, sur le fondement du mérite, pour établir des cercles concentriques de la citoyenneté et de la libertée ${ }^{48}$. Aux Amé-

47. La Bible est, bien sûr, mobilisée par les révolutionnaires, l'un des cas les plus importants étant celui de Thomas Paine dans son Common Sense (1776).

48. Claude-Olivier Doron, L'homme altéré. Races et dégénérescence (XVII -XIXe siècles), Seyssel, Champ Vallon, 2016. 
riques, ces enjeux étaient centraux puisqu'il fallut imaginer la liberté politique dans un contexte où de larges parties de la population ne jouissaient pas des droits accordés aux sujets liés généalogiquement à la métropole. Ce point dépasse, du reste, la question républicaine, puisque l'empire libéral du Brésil, après son indépendance, trancha le débat en ouvrant la citoyenneté politique aux libres de sang africain, pourvu qu'ils ne fussent pas nés en Afrique ${ }^{49}$.

\section{D’autres Atlantiques révolutionnaires, de Saint-Domingue à l'Amérique hispanique}

Le recentrement de la question républicaine autour des enjeux impériaux et coloniaux pose de fait l'hypothèse de "centres" qui ne seraient pas situés en Europe, ni constitués exclusivement par des Européens ou leurs descendants américains. La révolution des esclaves de Saint-Domingue et l'indépendance d'Haïti, depuis la thèse pionnière de Julius $S$. Scott ${ }^{50}$, a joué un rôle pilote dans ce décentrement à la fois méthodologique et politique. La révolution haïtienne révèle la part d'ombre et les non-dits des révolutions américaine et française, lesquelles, malgré la reconnaissance des droits naturels, n'ont pas touché à l'esclavage, dans le premier cas, ou ont échoué à l'abolir définitivement, pour l'autre. Dans cette histoire, ce n'est pas tant le républicanisme qui intéresse que l'impact d'une révolte d'esclaves qui se transforme en une révolution sociale et politique, pour aboutir à l'indépendance d'une nation. Le processus a souvent été lu, sans doute à tort, ainsi que l'a rappelé David Geggus $^{51}$, comme la construction d'une république noire, alors que Toussaint Louverture n'était pas républicain et qu'Haïti s'est divisé, après la mort de Dessalines (1807), en deux États dont l'un répudiait certes les rois, tandis que l'autre s'est défini comme un empire ${ }^{52}$, puis un royaume placé sous le sceptre de Christophe. Quoi qu'il en soit, les registres de la liberté et de l'égalité civile se sont largement diffusés dans le monde caribéen et au-delà, vers les côtes de l'Amérique du Nord, centrale et du Sud, via cet événement qui était à la fois une médiation de la Révolution française et le creuset d'une expérience politique inédite ${ }^{53}$. Aujourd'hui, cependant, la centralité prise par cet événement

49. Keila Grinberg, O fiador dos brasileiros: cidadania, escravidão e direito civil no tempo de Antonio Pereira Rebouças, Rio de Janeiro, Civilização Brasileira, 2002, et Gabriel Entin et Magdalena Candioti, «Liberté et dépendance pendant la révolution du Rio de la Plata. Esclaves et affranchis dans la construction d'une citoyenneté politique (1810-1820)", Le Mouvement Social, $\mathrm{n}^{\circ}$ 252, 2015, p. 71-91.

50. Julius Scott, The Common Wind. Currents of Afro-American Communication in the Era of the Haitian Revolution, PhD, Duke University, 1986; David B. Gaspar et David P. Geggus (eds), A Turbulent Time. The French Revolution and the Greater Caribbean, Bloomington, Indiana University Press, 1997.

51. David P. Geggus, "The Haitian Revolution in Atlantic Perspective", in Nicholas. Canny et Philip Morgan, (eds), The Atlantic World c. 1450-c. 1850, New York, Oxford University Press, 2011, p. 533-549.

52. Bernard Gainot, La révolution des esclaves, op. cit.

53. David Patrick Geggus, (ed.), The Impact of the Haitian Revolution in the Atlantic World, 
dans l'historiographie atlantique en vient à occulter d'autres expériences qui pourraient prétendre à constituer des nœuds révolutionnaires.

C'est, en réalité, dans l'Amérique ibérique que l'impact de SaintDomingue fut le plus important et le plus durable. Le précédent dominguois a fait réfléchir les futurs républicains et libéraux du sous-continent à l'un des enjeux centraux de la liberté civile : la création d'une société où la race ne serait plus un facteur structurant des hiérarchies juridiques et politiques. Il pousse les constituants vénézuéliens et colombiens à abolir, dès 1811, le principe de la pureté de sang qui organisait les hiérarchies raciales dans le monde hispanique depuis la fin du Moyen $\hat{A g e}^{54}$. Une forme embryonnaire d'internationalisme républicain joua, ici, son rôle : Bolívar, dont les armées étaient composées de libres de couleur et d'esclaves, trouva refuge et soutien auprès de la république du Sud d'Haïti, gouvernée par le président Pétion, en 1815-1816. Doté de subsides et soutenu par des marins et des soldats de l'île, le Libertador put reprendre le combat contre l'Espagne. Haïti demeura jusqu'en 1833 le seul État souverain qui fût abolitionniste dans le monde. Au Brésil, le soulèvement républicain du Pernambouc (1817) et les mouvements antimonarchistes qui menacèrent l'unité du pays à l'occasion de la révolution libérale portugaise de 1820, se sont référés aux symboles haïtiens. En 1835 , lors de la révolte des Malês - une insurrection d'esclaves musulmans à Bahia - certains chefs de la révolte portaient le portrait de Dessalines en pendentif55. Le motif se répète jusqu'à l'abolition de l'esclavage aux États-Unis en 1865 : Abraham Lincoln commence par obtenir la reconnaissance de la république noire en vue de l'adoption du XIIIe amendement ${ }^{56}$.

À ce décentrement s'ajoute la volonté de montrer la participation d'acteurs indigènes, métis ou d'origine africaine (libres ou esclaves) aux conflits politiques en tant qu'acteurs. Si l'histoire de ces sujets politiques commence à s'écrire, elle n'en constitue pas moins un fait capital et sous-estimé des révolutions républicaines de l'époque. Les premiers États indépendants de l'Amérique furent bâtis sur le sang de ces acteurs - qu'ils fussent républicains ou royalistes, puisque beaucoup choisirent ce dernier camp ${ }^{57}$. L'historiographie actuelle insiste sur le rôle actif et la politisation de ces groupes que

Columbia, University of South Carolina Press, 2001, et Alejandro Enrique Gómez Pernía, Le spectre de la révolution noire, op. cit.

54. Max S. Hering Torres, Maria Elena Martínez, David Nirenberg et (eds), Race and Blood in the Iberian World, Vienne, Berlin, Lit Verlag, 2012.

55. João José Reis, Slave rebellion in Brazil. Muslim Uprising of 1835 in Bahia, Baltimore, Johns Hopkins University Press, 1995.

56. Claire Bourhis-Mariotti, L'union fait la force. Les Noirs américains et Hä̈ti, 1804-1893, Rennes, Presses universitaires de Rennes, 2016.

57. Eric Van Young, The Other Rebellion. Popular Violence, Ideology, and the Mexican Struggle for Independence, 1810-1821, Stanford, Stanford University Press, 2001 ; Cecilia Méndez Gastelumendi, The Plebeian Republic. The Huanta Tebellion and the Making of the Peruvian State, 1820-1850, Durham, Duke University Press, 2005; Marcela Echeverri, Indian and Slave Royalists in the Age of Revolution. Reform, Revolution and Royalism in the Northern Andes, 1780-1825, Cambridge, Cambridge University Press, 2016. 
l'on décrivait naguère comme de la simple chair à canon. Le fait est patent à Saint-Domingue, à partir de la grande révolte des esclaves en 1791, mais il se vérifie lors de la Révolution américaine et en Amérique hispanique et portugaise $^{58}$.

Plusieurs courants de l'historiographie hispano-américaniste, d'inspirations très diverses, $\mathrm{y}$ insistent aujourd'hui à partir d'un terrain propice puisque les libres de couleur, les affranchis, les métis et les Amérindiens furent inclus constitutionnellement dans le cercle de la citoyenneté civile et politique dès l'émancipation de ces États ${ }^{59}$. Certains d'entre eux ont pratiqué un suffrage masculin quasi universel depuis leur création, comme les provinces argentines. Cette intégration par la loi est, bien entendu, loin d'avoir une traduction immédiate dans les pratiques politiques, puisqu'elle s'accompagne de divers procédés visant à subordonner ces nouveaux électeurs aux élites locales. Les "pathologies» du vote ont longtemps monopolisé l'attention, jusqu'aux années 1990 où l'historiographie a montré qu'il fallait prendre au sérieux la compétition électorale ${ }^{60}$. L'égalité civile et politique des libres eut le mérite de créer, dès le début du XIX ${ }^{\mathrm{e}}$ siècle, une arène où les groupes populaires ont revendiqué certaines formes de liberté et d'égalité dont beaucoup d'habitants européens ne jouissaient pas à la même époque ${ }^{61}$. Bien sûr, cet Atlantique politique de la liberté et de la révolution, largement républicain, accompagne celui de la servitude et de la contre-révolution, puisque le $\mathrm{XIX}^{\mathrm{e}}$ siècle représente le point culminant de la traite transatlantique, surtout vers Cuba et le Brésil.

Une telle remise en question importe puisqu'il s'agit de penser la construction des formes modernes d'émancipation politique et sociale par des acteurs non-européens ou métis, à la fois hors de la matrice nord-atlantique et en lien avec elle. Elle incite à considérer avec attention le rôle d'espaces sociologiquement et culturellement hybrides, alliant ou opposant des élites d'origine européenne, mais pas toujours, à des groupes populaires et métis dans ces processus politiques. Cette perspective invite à décentrer l'histoire de la république, à la "déprovincialiser" en somme, du point de vue de ses registres intellectuels et de ses répertoires d'actions. Ce programme ne se résume pas à la critique facile de l'européocentrisme; c'est aussi une façon de remettre en question l'opposition entre la modernité européenne et ce qui serait sa négation, son Autre ou son envers, pour repenser cette relation de façon

58. Hendrik Kraay, Race, State, and Armed Forces in Independence-Era Brazil. Bahia, 1790s-1840s, Stanford, Stanford University Press, 2004.

59. Hebe Maria Mattos de Castro, Escravidão e cidadania no Brasil monárquico, Rio de Janeiro, Jorge Zahar, 2000; Keila Grinberg, O fiador dos brasileiros: cidadania, escravidão e direito civil no tempo de Antonio Pereira Rebouças, Rio de Janeiro, Civilização Brasileira, 2002.

60. Antonio Annino [dir.], Historia de las elecciones en Iberoamérica, siglo XIX, Buenos Aires, Fondo de Cultura Económica, 1995.

61. Hilda Sabato, Republics of the New World. The Revolutionary Political Experiment in Nineteenth-Century Latin America, Princeton, Princeton University Press, 2018. 
plus large et plus complexe ${ }^{62}$. Cet Atlantique politique précise ses contours à l'âge des révolutions, même s'il se construit plus tôt. Il maintient sa relative cohérence jusqu'à la fin du XIX ${ }^{\mathrm{e}}$ siècle, au moins, grâce aux parcours de milliers de volontaires, militaires, militants exilés ou bannis, et aux répertoires communs permettant de donner sens aux enjeux politiques nationaux et transnationaux, avec leurs étiquettes unifiantes : droit naturel, constitutionnalisme, abolitionnisme, républicanisme et libéralisme, puis socialisme et anarchisme ${ }^{63}$.

Remarquons cependant que, entendu ainsi, le décentrement que suppose une lecture des républicanismes au niveau de l'Atlantique reste partiel. La prise en compte des Amériques et de quelques exemples africains, tous liés aux circuits de la traite et aux besoins du commerce, excluent, de fait, les configurations culturelles, religieuses, politiques qui ne doivent rien à l'Europe. À ma connaissance, les communautés politiques africaines ou amérindiennes indépendantes sont encore peu présentes au menu des enquêtes historiques en cours sur les révolutions atlantiques. Il faudrait pourtant mieux comprendre comment s'articulent les formes "occidentales" de la communauté avec les corps politiques autochtones d'Afrique et d'Amérique ${ }^{64}$. Certains historiens, comme Sinclair Thomson, proposent, par exemple, d'inclure les grandes révoltes amérindiennes des années 1780-1782, comme celle de Tupac Amaru au Pérou, dans la réflexion sur l'âge des révolutions, même si leurs revendications à propos de la construction des souverainetés indigènes ne s'inscrivaient pas dans les répertoires politiques des autres moments révolutionnaires ${ }^{65}$. Les travaux ont ainsi avancé concernant l'Amérique espagnole, où l'on a pu montrer que beaucoup de communautés amérindiennes, redéfinies par leur intégration tri-séculaire à la monarchie espagnole, avaient rapidement digéré le «libéralisme» de la constitution libérale de Cadix, par exemple, pour utiliser certaines de ses dispositions à leur avantage ${ }^{66}$. D'autres ont souligné leur capacité à s'approprier les valeurs républicaines pour défendre leurs droits collectifs ou les accroître. L'une des tâches futures de l'historiographie pour-

62. Dipesh Chakrabarty, Provincializing Europe. Postcolonial Thought and Historical Difference, Princeton, Princeton University Press, 2012, introduction, passim, traduction française Provincialiser l'Europe. La pensée postcoloniale et la différence historique, Paris, Amsterdam, 2009.

63. Nous nous permettons de signaler María Teresa Calderón et Clément Thibaud [dir.], Las revoluciones en el mundo atlántico, Bogotá, Madrid, Taurus, 2006; Federica Morelli, Clément Thibaud et Geneviève Verdo [dir.], Les empires atlantiques des Lumières au libéralisme (1763-1865), Rennes, Presses universitaires de Rennes, 2009; Clément Thibaud, Gabriel Entin, A. Gómez Pernía et Federica Morelli [dir.], L’Atlantique révolutionnaire. Une perspective ibéro-américaine, Bécherel, Les Perséides, 2013.

64. Voir, a contrario, le beau livre d'Adrien Delmas et Nigel Penn (dir.), Written Culture in a Colonial Context. Africa and the Americas, 1500-1900, Le Cap, UCT Press, 2013.

65. Sinclair Thomson, We Alone Will Rule. Native Andean Politics in the Age of Insurgency, Madison, University of Wisconsin Press, 2002.

66. Un exemple récent : Bartolomé Clavero, «Régimen de misiones y autonomía indígena: doble cara de Cádiz en Nueva Granada» et Victor Peralta Ruiz, "De la persistencia al olvido. La impronta constitucional gaditana en el Perú del siglo XIX», in Maria Teresa Calderón [dir.], Política y constitución en tiempos de las independencias, Bogotá, Universidad Externado de Colombia, 2017, respectivement p. 157-178 et p. 391-410. 
rait consister à intégrer ces expériences dans la trame d'une histoire politique «à parts égales» ${ }^{67}$.

\section{De l’Atlantique À la Méditerranée}

Ce décentrement vers les Amériques ne doit pas faire pour autant oublier l'Europe. Si les indépendances américaines - du Nord et du Sud - furent des révolutions à la dimension de l'Atlantique, il est légitime de se demander quels furent leurs effets en retour sur leurs anciennes métropoles et au-delà. Cette question paraît d'emblée plus pertinente si l'on sort un moment de la thématique strictement républicaine pour s'attacher aux circulations libérales qui ont pu lier les deux rives de l'océan (sachant, néanmoins, que ces deux répertoires étaient enchevêtrés outre-Atlantique, comme on l'a vu). La décennie 1820 apparait en tout point comme décisive dans ce basculement politique de l'Atlantique vers l'Europe du Sud et la Méditerranée ${ }^{68}$. Du côté des Ibériques, les révolutions libérales de 1820, en Espagne et au Portugal, prennent tout leur sens à l'échelle impériale et atlantique, tant et si bien que Gabriel Paquette a pu évoquer la guerre civile portugaise de 1828-1834 comme étant la dernière des « révolutions atlantiques ${ }^{69}$ ». Des travaux récents ont permis d'éclairer la constitution d'une «internationale libérale» dans les pays du Sud du vieux continent ${ }^{70}$. Composée d'exilés et de bannis venus d'Italie, d'Espagne ou du Portugal, ce groupe, dont on ne sait s'il a formé un réseau ou une diaspora politique, doit sa cohérence à son adhésion aux valeurs constitutionnelles déposées dans la constitution de Cadix (1812) et aux persécutions des régimes de restauration, comme celui de Ferdinand VII en Espagne. Ces constitutionnels modérés, pour la plupart monarchistes, ont lutté, en tant que volontaires, pour la Grèce indépendante ou même en faveur des émancipations hispano-américaines ${ }^{71}$. Ils constituent l'un des groupes le plus nombreux qui s'oppose aux valeurs du Congrès de Vienne. Il faut y ajouter, bien sûr, les marins en rupture de ban, les anciens de l'Empire et les exilés de l'Europe de Vienne ${ }^{72}$.

67. Cf. Elizabeth Mancke, "Polity formation and atlantic political narratives", in Nicholas Canny et Philip Morgan (eds), The Oxford Handbook of the Atlantic World, Oxford, Oxford University Press, 2011, p. 382-399.

68. Matthew Brown et Gabriel Paquette (eds), Connections After Colonialism. Europe and Latin America in the 1820s, Tuscaloosa, University of Alabama Press, 2013.

69. Gabriel B. Paquette, Imperial Portugal in the Age of Atlantic Revolutions. The Luso-Brazilian World, c. 1770-1850, Cambridge, Cambridge University Press, 2013, chapitre 4.

70. Un dossier ouvert par Jeanne Moisand [dir.], «Exils et circulations des idées politiques entre Amérique hispanique et Espagne après les indépendances (1820-1836) ", Revue d'histoire du XIXe siècle, 2015, n $^{\circ} 51$, p. 35-51.

71. Delphine Diaz, Un asile pour tous les peuples. Exilés et réfugiés étrangers dans la France du premier XIXe siècle, Paris, Armand Colin, 2014.

72. Walter Bruyère-Ostells, La grande armée de la liberté, Paris, Tallandier, 2014; Nicolas Terrien, Des patriotes sans patrie. Histoire des corsaires insurgés de l'Amérique espagnole, Mordelles, Les Perséides, 
Beaucoup ont trouvé refuge, à un moment ou à un autre de leur trajectoire politique, de l'autre côté de l'océan, aux États-Unis, certes, mais aussi au Mexique, au Brésil, en Uruguay, dans les provinces argentines. Les contours de cette émigration politique sont difficiles à tracer pour deux raisons. La première est l'évolution politique des individus qui composent ces cohortes, souvent fluctuantes et conditionnées par les contextes sociopolitiques dans lesquels ils évoluent. La seconde tient au caractère hétérogène des opinions et des expériences de chacun. Ceux qui ont été compromis dans les régimes des républiques-sœurs, dans la péninsule italienne par exemple, ou par leur adhésion à l'Empire napoléonien, comme les Afrancesados espagnols ${ }^{73}$, ne ressemblent pas aux libéraux modérés que l'intransigeance de certaines restaurations a conduits sur le chemin de l'exil. Cette nébuleuse gyrovague, qui va de la charbonnerie aux libéraux de la péninsule ibérique ${ }^{74}$ en passant par Giovane Italia, commence à être bien connue ${ }^{75}$. Maurizio Isabella et Konstantina Zanou proposent d'étendre les limites de cette Méditerranée révolutionnaire, cantonnée à sa rive nord, à l'espace politique incluant les ports du Levant, du Maghreb et de l'Égypte ${ }^{76}$. L'enjeu serait ainsi de mieux montrer l'articulation entre cette Méditerranée politique et le continent américain.

Cela constitue l'une des conditions nécessaires pour essayer de mieux comprendre comment les réseaux de ces bannis, exilés ou militants, avant tout définis par leur adhésion au constitutionnalisme libéral, exploitent les ressources politiques ouvertes par l'existence d'un Atlantique républicain. D’un point de vue chronologique, géographique et thématique, des enquêtes ont commencé à explorer cet espace politique qui émerge avec l'âge des révolutions, puis se consolide et se diversifie tout au long du XIX ${ }^{\mathrm{e}}$ siècle ${ }^{77}$. Certes, peu d'entre elles abordent les circulations républicaines en tant que telles, si ce n'est en s'attachant aux capitales de l'exil transatlantique que furent Philadelphie $^{78}$, New York, Londres, Paris, Bruxelles. Mais on sait très peu de chose sur le rôle de nombreux exilés politiques hispano-américains en Europe, parmi lesquels figuraient des personnages éminents. L'enquête doit

2015; Nathan Perl-Rosenthal, Citizen Sailors. Becoming American in the Age of Revolution, Cambridge (Mass.), Harvard University Press, 2015.

73. Jean-René Aymes, Los españoles en Francia, 1808-1814. La deportación bajo el Primer Imperio, Madrid, Siglo XXI, 1987.

74. Juan Luis Simal, Emigrados. España y el exilio internacional, 1814-1834, Madrid, Centro de Estudios Políticos y Constitucionales, 2012.

75. Cf. Éric Anceau, Jacques-Olivier Boudon et Olivier Dard [dir.], Histoire des internationales. Europe, XIXe-XXe siècles, Paris, Nouveau Monde, 2017; Maurizio Isabella, Risorgimento in Exile. Italian Emigrés and the Liberal International in the Post-Napoleonic Era, Oxford, Oxford University Press, 2009.

76. Maurizio Isabella et Konstantina Zanou (eds), Mediterranean Diasporas. Politics and Ideas in the long $19^{\text {th }}$ Century, London, Bloomsbury, 2016.

77. Delphine Diaz, Jeanne Moisand, Romy Sánchez et Juan Luis Simal [dir.], Exils entre les deux mondes. Migrations et espaces politiques atlantiques au XIX e siècle, Mordelles, Les Perséides, 2015; Romy Sánchez, Quitter la Très fidèle..., op. cit.; thèse en cours de Clara Avendaño sur L'immigration politique sud-américaine en Europe dans la première moitié du XIXe siècle (Université Paris-Sorbonne).

78. Monica Henry, «Les premières publications révolutionnaires des exilés hispano-américains aux États-Unis », Transatlantica, 2, 2006 : http://transatlantica.revues.org/1146 
aussi porter sur les bannis de l'Europe légitimiste, dont beaucoup ont trouvé refuge aux Amériques ${ }^{79}$. La colonie agraire du Champ-d'Asile, fondée en 1818 au Texas, regroupait ainsi des exilés de toutes les nationalités, liés par la compromission avec les régimes napoléoniens en Europe. Elle préfigure d'autres «républiques» éphémères, comme les colonies phalanstériennes ou communistes d'Étienne Cabet ou Victor Considerant ${ }^{80}$, mais leur existence, le plus souvent fugace, témoigne de la possibilité d'expériences politiques visant la construction de communautés de travail autonomes ${ }^{81}$. Celles-ci ont profité d'espaces de frontières, comme le Texas, la Californie ${ }^{82}$ et plusieurs régions côtières de l'Amérique centrale. Ces circulations politiques accompagnent le déploiement progressif de la colonisation européenne de masse (settler colonization ${ }^{83}$ ), où la mise en valeur des terres américaines par des colons pauvres apparaît comme une solution à la question sociale européenne, tout en "améliorant la race» dans le nouveau monde.

L'échelle d'analyse atlantique permet d'isoler quelques grands traits du républicanisme au XIX ${ }^{e}$ siècle : d'abord américain, anti-impérial, il prospère, contre toute attente, au sein de sociétés marquées par l'esclavage et la racialisation, mais aussi très profondément religieuses et peu ou pas sécularisées ${ }^{84}$. Et c'est dans ces espaces sociaux a priori bien éloignés des répertoires républicains que des régimes antimonarchiques ont prospéré. C'est, sans aucun doute, l'opposition à la forme impériale, qu'elle fût coloniale ou pas, qui explique la floraison de ces nations sans roi. La rupture avec les métropoles européennes se fit contre le "despotisme" et l'exclusif, conduisant à l'association précoce du républicanisme avec certains registres libéraux. La constitution d'États indépendants contre la centralité impériale et la verticalité métropolitaine a encouragé ces nations à lier cités et provinces grâce à la manœuvre fédérale, qui a, de ce fait, marqué durablement la pensée et les pratiques républicaines de l'époque, même en France.

Dans ce contexte, le paradigme de la Révolution atlantique, celle de Jacques Godechot et de Robert Palmer, a oublié les enjeux de l'esclavage et de la race. C'est pourtant sur le continent américain - en y ajoutant deux expériences africaines de signes contraires, le Libéria et les républiques boers -

79. Erwann Terrien, The Wild and Extravagant Projects of these French Fugitives. A Transatlantic Study of the Myths and Realities of Champ d'Asile (1818-1820), PhD, The University of Texas, 2011.

80. Michel Cordillot, Utopistes et exilés du Nouveau Monde. Des Français aux Etats-Unis, de 1848 à la Commune, Paris, Vendémiaire, 2013.

81. Laurent Vidal, Ils ont rêvé d'un autre monde, Paris, Flammarion, 2014.

82. Emmanuelle Pérez-Tisserant, Nuestra California. Faire Californie entre deux constructions nationales et impériales (vers 1810-1850), thèse d'histoire sous la direction de François Weil, EHESS, 2014.

83. James Belich, Replenishing the Earth. The Settler Revolution and the Rise of the Anglo-world, 1783-1939, Oxford, Oxford University Press, 2011.

84. Développer ce point fondamental pourrait faire l'objet d'un autre article. 
que les régimes et les mobilisations antimonarchiques fleurirent tout au long du XIX ${ }^{\mathrm{e}}$ siècle. Même si, à l'origine, beaucoup d'États n'eurent pas l'intention d'abolir l'esclavage, et si la communauté des égaux s'y limita souvent, en fait ou en droit, à celle des descendants d'Européens, elles furent aussi le théâtre de combats continus pour la liberté, l'égalité ou la terre. C'est pourquoi, dès la fin du XVIII siècle, l'histoire de la république démocratique intéresse aussi les luttes autour de l'esclavage, de la race ou du statut des Amérindiens. Si ces acteurs n'étaient pas nécessairement républicains, ni ne se référaient toujours à ces répertoires, ils ont profondément modelé le républicanisme comme forme de régime et comme répertoire de valeurs tout au long du XIX ${ }^{\mathrm{e}}$ siècle.

Il serait intéressant, pour finir, de relire l'histoire convulsée des républiques européennes du XIX ${ }^{\mathrm{e}}$ siècle à l'aune de ce que nous dit cet «âge des révolutions" centré sur l'Amérique. Cause commune des républiques américaines, la chute des empires européens de l'Atlantique a produit des registres de pratiques et d'idées (constitutionnelles, républicaines, libérales) dont on soupçonne qu'elles furent réinvesties ailleurs, dans la critique d'autres empires pluriséculaires - ottoman, qadjar, chinois -, sans parler des parties asiatiques et africaines des souverainetés impériales de l'Europe.

Clément Thibaud est directeur d'études de l'EHESS, Mondes Américains-UMR 8168 